\title{
Effect of high-fat diet on peripheral blood mononuclear cells and adipose tissue in early stages of diet-induced weight gain
}

\author{
Jake E. Lowry ${ }^{1} \dagger$, Batbayar Tumurbaatar ${ }^{2} \dagger$, Claudia D’Agostino ${ }^{1}$, Erika Main ${ }^{1}$, Traver J. Wright ${ }^{2}$, \\ Edgar L. Dillon ${ }^{2}$, Tais B. Saito ${ }^{3,4}$, Craig Porter ${ }^{5,6}$, Clark R. Andersen ${ }^{7}$, Douglas L. Brining ${ }^{1}$, Janice J. Endsley ${ }^{3}$, \\ Melinda Sheffield-Moore ${ }^{2}$, Elena Volpi ${ }^{2}$, Rong Fang ${ }^{4}$, Nicola Abate ${ }^{2}$ and Demidmaa R. Tuvdendorj ${ }^{2 *}$ \\ ${ }^{1}$ Animal Resource Center, University of Texas Medical Branch, Galveston, TX 77550, USA \\ ${ }^{2}$ Department of Internal Medicine, University of Texas Medical Branch, Galveston, TX 77550, USA \\ ${ }^{3}$ Department of Microbiology \& Immunology, University of Texas Medical Branch, Galveston, TX 77550, USA \\ ${ }^{4}$ Department of Pathology, University of Texas Medical Branch, Galveston, TX 77550, USA \\ ${ }^{5}$ Department of Surgery, University of Texas Medical Branch, Galveston, TX 77550, USA \\ ${ }^{6}$ Metabolism Unit, Shriners Hospital for Children, Galveston, TX 77550, USA \\ ${ }^{7}$ Preventive Medicine \& Community Health, University of Texas Medical Branch, Galveston, TX 77550, USA
}

(Submitted 18 July 2019 - Final revision received 3 September 2019 - Accepted 22 September 2019)

\section{Abstract}

Subcutaneous adipose tissue (scAT) and peripheral blood mononuclear cells (PBMC) play a significant role in obesity-associated systemic lowgrade inflammation. High-fat diet (HFD) is known to induce inflammatory changes in both scAT and PBMC. However, the time course of the effect of HFD on these systems is still unknown. The aim of the present study was to determine the time course of the effect of HFD on PBMC and scAT. New Zealand white rabbits were fed HFD for 5 or 10 weeks (i.e. HFD-5 and HFD-10) or regular chow (i.e. control (CNT)- 5 and CNT-10). Thereafter, metabolic and inflammatory parameters of PBMC and scAT were quantified. HFD induced hyperfattyacidaemia in HFD-5 and HFD10 groups, with the development of insulin resistance in HFD-10, while no changes were observed in scAT lipid metabolism and inflammatory status. HFD activated the inflammatory pathways in PBMC of HFD-5 group and induced modified autophagy in that of HFD-10. The rate of fat oxidation in PBMC was directly associated with the expression of inflammatory markers and tended to inversely associate with autophagosome formation markers in PBMC. HFD affected systemic substrate metabolism, and the metabolic, inflammatory and autophagy pathways in PBMC in the absence of metabolic and inflammatory changes in scAT. Dietary approaches or interventions to avert HFD-induced changes in PBMC could be essential to prevent metabolic and inflammatory complications of obesity and promote healthier living.

Key words: Obesogenic diet: Weight gain: Low-grade inflammation: Autophagy: Peripheral blood mononuclear cells: Subcutaneous adipose tissue

Obesity results in systemic and chronic low-grade inflammation with an increased number of circulating immune cells and inflammatory cytokines ${ }^{(1-3)}$. This inflammation is associated with worsening metabolic health and the development of obesityassociated chronic diseases. Thus, understanding the mechanisms whereby systemic low-grade inflammation develops and determining approaches to prevent its progression should help promote or prolong healthier living. Currently, subcutaneous and visceral adipose tissue (scAT and VAT, respectively) inflammation is suggested as the main cause for the development of systemic low-grade inflammation ${ }^{(4-6)}$. However, Tan et al. ${ }^{(7)}$ demonstrated that 28-d overfeeding led to the development of systemic insulin resistance (IR) and increased the concentration of circulating C-reactive protein and monocyte chemoattractant protein-1. Interestingly, these effects were observed in the absence of changes in scAT adipocyte size or the proportion of macrophages and T-cell populations. Moreover, overfeeding-associated expansion of VAT directly correlated with the inability of scAT to

Abbreviations: ASR, absolute synthesis rate; CNT, control; FAO, fatty acid $\beta$-oxidation; FSR, fractional synthesis rate; HFD, high-fat diet; HOMA-IR, homeostatic model assessment of insulin resistance; IR, insulin resistance; MAPK, mitogen-activated protein kinase; PBMC, peripheral blood mononuclear cells; RA, rate of appearance; scAT, subcutaneous adipose tissue; TLR4, toll-like receptor 4; VAT, visceral adipose tissue.

* Corresponding author: Demidmaa R. Tuvdendorj, fax +409772 8709, email detuvden@utmb.edu

$\dagger$ These authors contributed equally to this work. 
accumulate lipids $^{(8)}$. These results suggest that, in the early stages of overfeeding, the systemic inflammation may associate with activation of inflammatory mechanisms in cells other than adipocytes.

Circulating immune cells are also known to be involved in obesity-associated systemic low-grade inflammation. The effect of nutrition on peripheral blood mononuclear cells (PBMC) has been a subject of increased interest. Food intake has been shown to transiently induce oxidative stress and pro-inflammatory changes in the PBMC of healthy individuals ${ }^{(9)}$. The plasma concentration of NEFA is known to significantly increase the expression of IL- 6 and TNF- $\alpha$ mRNA in PBMC ${ }^{(1)}$. These data show that PBMC have a dynamic inflammatory response to nutrient intake. However, not much is known about the long-term effect of diet. This information is critically important, as currently most of the humans spend a considerable amount of time in a non-fasting, postprandial state with hyperlipidaemia ${ }^{(10,11)}$. This state may promote chronic activation of inflammatory pathways in PBMC, which may precede and/or drive the inflammatory changes in scAT. Moreover, in vitro studies have demonstrated that the common dietary SFA palmitate can activate autophagy pathways $^{(12)}$ in PBMC, which also play a significant role in monocyte-macrophage differentiation ${ }^{(13)}$ and systemic low-grade inflammation. However, no information is available if high-fat diet (HFD) affects autophagy pathways in circulating immune cells in vivo. Therefore, we aimed to test our hypothesis whether increased dietary fat intake activates inflammatory and autophagy pathways in vivo in PBMC prior to metabolic and inflammatory changes in scAT. We also aimed to determine the underlying mechanisms and the time-course effect of HFD (i.e. 5- and 10week dietary interventions) on activation of these pathways.

\section{Methods}

\section{Animals and dietary interventions}

Male New Zealand white rabbits (Crossroads Rabbitry) were used for the present study. We chose this model because first, previous studies have shown that the lipid metabolism in this animal model of diet-induced obesity resembles that of humans with obesity ${ }^{(14)}$. Second, the size of this animal model allows us to conduct stable isotope tracer infusion studies and to obtain the required samples from one animal to achieve the objectives of our studies ${ }^{(14,15)}$. All animals were acclimatised for 1-2 weeks before being randomly assigned to study groups. Twelve rabbits were assigned into 5- and 10-week dietary intervention with HFD (i.e. HFD-5 and HFD-10 groups, respectively). Upon completion of the intervention, all animals underwent a metabolic in vivo study as described below. Twelve control (CNT) animals, age matched with HFD- 5 and HFD-10 groups, and denoted as CNT-5 and CNT-10 groups, underwent the same metabolic in vivo study. Thus, the four groups of animals included HFD-5 ( $n$ 6), HFD-10 ( $n$ 6), CNT-5 ( $n$ 6), and CNT-10 ( $n$ 6). Animals in the CNT groups were fed Laboratory Rabbit High Fiber diet (catalogue no. 5326; Labdiet ${ }^{\circledR}$ ) ad libitum. Animals in the HFD groups were fed the same diet plus additional $10 \%$ maize oil and $8 \%$ lard, as previously described ${ }^{(15)}$. The compositions of diets are presented in supplemental files. All animals were individually housed with light/dark cycle and social interaction. The welfare-related assessments were performed throughout the study duration by the veterinary personnel of the Animal Research Center, the University of Texas Medical Branch (UTMB) at Galveston. The study protocol complied with the Public Health Service Policy on Humane Care and Use of Laboratory Animals, incorporated in the Institute for Laboratory Animal Research Guide for Care and Use of Laboratory Animals and was approved by the Animal Care and Use Committee of UTMB.

\section{The metabolic in vivo study}

Following the dietary intervention and after an overnight fast (about $12 \mathrm{~h}$ ), all animals were subjected to a metabolic study. In the morning of the study, animals were anesthetised with ketamine and xylazine, and fur shaved on the chest, neck and one ear. Jugular and marginal ear veins and the carotid artery were cannulated along with intubation of the trachea or placement of a laryngeal airway mask and ventilated (rate $12 \mathrm{bpm}$, volume $100 \mathrm{ml}$ pressure not to exceed $20 \mathrm{mmHg}$ ). Venous blood (about $20 \mathrm{ml}$ ) was collected in EDTA vacutainer tubes and abdominal scAT and skeletal muscle samples from vastus lateralis were also collected. Thereafter, a $3-\mathrm{h}$ primed continuous infusion of $\mathrm{U}-\left[{ }^{13} \mathrm{C}_{16}\right]$-palmitate $(99 \%$ enriched; Cambridge Isotope Laboratories, Inc.) in $5 \%$ albumin (priming dose: $1.0 \mu \mathrm{mol} / \mathrm{kg}$, infusion rate: $0 \cdot 1 \mu \mathrm{mol} / \mathrm{kg}$ per $\mathrm{min}$ ) was started ${ }^{(15,16)}$. About $3 \mathrm{ml}$ of arterial blood was obtained at 30, 60, 90, 120, 150, 160,170 and $180 \mathrm{~min}$ of infusion to determine the rate of appearance (Ra) of palmitate as a measure of the lipolysis rate. Thereafter, the animals were killed by intravenous injection of $5 \mathrm{ml}$ of Euthasol solution under general anaesthesia using ketamine and xylazine. Death was confirmed by open chest observation. At this time, a liver sample was obtained.

\section{Ex vivo studies}

Ex vivo studies were designed to determine the rate of incorporation of $\mathrm{U}-\left[{ }^{13} \mathrm{C}_{16}\right]$-palmitate into palmitoyl-carnitine under a basal condition as a marker of mitochondrial fatty acid $\beta$-oxidation (FAO) ${ }^{(16)}$. About $4 \mathrm{ml}$ of baseline blood sample collected in EDTA-containing tubes was used. After obtaining samples to measure the background parameters (i.e. the enrichment of $\mathrm{U}-\left[{ }^{13} \mathrm{C}_{16}\right]$-palmitate and the total concentrations of NEFA and acylcarnitines), the remaining samples $(3.6 \mathrm{ml})$ were mixed with $1 \mu \mathrm{l} / \mathrm{ml}$ of $2 \mathrm{~mm} \mathrm{U-}\left[{ }^{13} \mathrm{C}_{16}\right]$-palmitate dissolved in $5 \%$ human albumin. The samples were then incubated in $37^{\circ} \mathrm{C}$ water bath with periodic mixing, and $(0.4 \mathrm{ml})$ aliquots were collected at 5,10 , 20, 40 and $60 \mathrm{~min}$ after the start of incubation. All aliquots were immediately frozen in liquid $\mathrm{N}_{2}$ to arrest all biochemical reactions.

\section{Sample analyses}

Glucose and insulin measurements. Blood glucose levels were measured using an Ascensia glucometer (Bayer). Plasma insulin concentrations were measured using ELISA kits (Mercodia). 
Plasma NEFA. Plasma lipids were extracted using a heptanepropanol extraction buffer, and NEFA were separated using TLC plates (Partisil LK5D, Silica Gel 150 A; Schleicher \& Schuell). After the samples were methyl-esterified, the tracer: tracee ratio of $\mathrm{U}_{-}\left[{ }^{13} \mathrm{C}_{16}\right]$-palmitate in plasma NEFA was measured using GC-MS (MSD system; Agilent), monitoring the mass:charge ratios of 270, 285 and 286 for methyl palmitate. Eight fatty acids in plasma NEFA were measured using a GC system with flame ionisation detection (GC-FID 6890; Agilent); the total NEFA concentrations were measured by means of internal standards using a GC-FID system ${ }^{(15,16)}$

Analyses of blood lipids and acylcarnitines from ex vivo experiments. Blood NEFA were isolated and analysed as described above. Blood acylcarnitines were isolated using $1 \mathrm{ml}$ of an acetonitrile-methanol mixture (3:1) with $50 \mu \mathrm{l}$ of $1 \mathrm{~m}$ $\mathrm{KH}_{2} \mathrm{PO}_{4}{ }^{(14,15)}$. To measure the concentrations of specific and total acylcarnitine species, $\mathrm{D}_{3}$-C16:0-carnitine was added as an internal standard $(45 \mu \mathrm{m})$. Samples were then analysed to determine the concentrations of myristoyl-, palmitoyl- and total acylcarnitines using an LC-MS (LC-MS 6130 Quadruple; Agilent), as previously described ${ }^{(15,16)}$. The enrichment of $\mathrm{U}_{-}\left[{ }^{13} \mathrm{C}_{16}\right]$-palmitoyl-carnitine was measured to determine the tracer:tracee ratio of the label incorporated into palmitoyl-carnitine.

Muscle and liver lipid analyses. The lipids were extracted from 30 to $50 \mathrm{mg}$ of tissue powder overnight at $4^{\circ} \mathrm{C}$ in a $1: 2(\mathrm{v} / \mathrm{v})$ methanol-chloroform solution containing $0.05 \mathrm{mg} / \mathrm{ml}$ butylated hydroxytoluene. The samples were subjected to a TLC plate to separate TAG and NEFA, as previously described ${ }^{(15-17)}$. The TAG and NEFA profiles were determined using GC-FID, and the contents were calculated using internal standard approach, as previously described ${ }^{(15,18)}$

Western blot analyses. The PBMC were isolated from baseline samples using Ficoll gradient separation as previously described $^{(18)}$. scAT proteins were isolated as previously described $^{(17)}$. The protein extracts of PBMC cytosolic fraction, and of scAT $(15 \mu \mathrm{g})$, were subjected to $4-15 \%$ SDS-PAGE (Criterion TGX; Bio-Rad Laboratories) and electrophoretically transferred to polyvinylidene difluoride membranes (Immobilon ${ }^{\circledR}$-P; Millipore). The following monoclonal primary antibodies were used: (a) t-p38 (no. 9212S; 1:1000; $38 \mathrm{kDa}$ ), p-p38 (no. 4511; 1:1000; $38 \mathrm{kDa}$ ), t-ERK1/2 (no. 9102; 1:100; 42/44 kDa), p-ERK1/2 (no. 9910T; 1:100; 42/44 kDa), LC3 (no. 4445; 1:1000; 14/16 kDa), p62 (no. 4445; 1:500; $62 \mathrm{kDa}$ ), t-perilipin (no. 9349; 1:1000; $57 \mathrm{kDa}$ ) from Cell Signaling; (b) IL-1 $\beta$ (sc32294; 1:200; $17 \mathrm{kDa}$ ), IL-10 (sc8438; 1:200; $20 \mathrm{kDa}$ ), toll-like receptor 4 (TLR4) (sc-293072; 1:1000; $95 \mathrm{kDa}$ ) from Santa Cruz; (c) p-perilipin ${ }^{\text {Ser522 }}$ (no. 4856; 1:5000; $57 \mathrm{kDa}$; ValaSciences) and (d) adiponectin (ab22554; 1:1000; $30 \mathrm{kDa}$; Abcam). As a secondary antibody, either goat anti-mouse IgG-horseradish peroxidase (HRP) (no. 1030-05, 1:5000-10 000) or anti-rabbit IgG-HRP (no. 4050-05; 1:5000-10 000; Southern Biotech) was used. The expression levels were evaluated by quantification of the relative density of each band normalised to the $\beta$-actin band density (no. A1978, 1:50 000, 42 kDa; Sigma-Aldrich) using National Institutes of Health (NIH) ImageJ software v1.46r (NIH).

\section{Calculations}

IR (homeostatic model assessment of insulin resistance (HOMAIR)) and $\beta$-cell function (HOMA- $\beta \%$ ) were estimated from fasting glucose and insulin values using the approximated equation of Matthews et $a l^{(19)}$ and the disposition index, estimated as HOMA- $\beta \% /$ HOMA-IR ${ }^{(20)}$.

The expression of IL- $1 \beta$ was divided by the expression of IL-10 (arbitrary units) to calculate the ratio of IL-1 $\beta$ :IL-10 as a marker of distribution of activated (M1-type) $v$. classical (M2type) immune cells ${ }^{(21)}$.

The $\mathrm{Ra}$ of palmitate, a measure of in vivo lipolysis rate, was calculated by dividing the enrichment of $\mathrm{U}-\left[{ }^{13} \mathrm{C}_{16}\right]$-palmitate in plasma NEFA by rate of infusion of $\mathrm{U}_{-}\left[{ }^{13} \mathrm{C}_{16}\right]$-palmitate ${ }^{(16)}$. For ex vivo studies, to calculate the kinetic parameters, the tracer: tracee ratios of labelled palmitic acid and palmitoyl-carnitine were converted into molar percentage excess (MPE). The fractional synthesis rate (FSR) of palmitoyl-carnitine was calculated by dividing the slope of incorporation of the label into palmitoylcarnitine by the AUC of labelled palmitate (MPE) over a 1-h ex vivo experimental period and is expressed as $\% / \mathrm{h}$. The absolute synthesis rate (ASR) of palm-carnitine was calculated by multiplying the FSR by the concentration of palmitoyl-carnitine and expressed as $\mathrm{nmol} / \mathrm{l}$ per $\min ^{(16)}$.

Statistical analyses and data presentation. Data are presented as means and standard errors. The sample size was determined by the feasibility of minimally detectable effect sizes. The differences in parameters between the groups were evaluated using a two-way ANOVA with the factor's diet (i.e. CNT or HFD) and time (i.e. 5 or 10 weeks) with post hoc Tukey correction. The approximate normality of model residuals was verified using normal quantile plots. Pairwise correlations between palmitoyl-carnitine FSR select outcomes of interest were determined using linear regression. $P<0.05$ was considered statistically significant.

\section{Results}

\section{Systemic and tissue metabolism}

All results are presented in Table 1 . HFD of 5 and 10 weeks resulted in a gain of 0.65 ( $\mathrm{sE} 0.16$ ) and 1.19 ( $\mathrm{SE} 0.83$ ) kg of body weight from the baseline, respectively. However, no significant differences in body weight were observed between the CNT and HFD groups at either time point. HFD significantly increased the plasma concentration of NEFA at both time points; while the lipolysis rate $(\mathrm{Ra})$ only trended to decline over time. Western blot analyses of scAT samples confirmed these data, demonstrating that although the expression of total perilipin was significantly lower in HFD-10, the phosphorylated perilipin:total perilipin ratio was not altered (Fig. 1, Table 1). The expression of adiponectin and TLR 4 as markers of inflammation in scAT did not differ between the HFD and CNT groups (Fig. 1, Table 1). Furthermore, liver and muscle lipid contents were not significantly altered by HFD

Although not reaching statistical significance, HFD-5 averaged 24 and $81 \%$ higher plasma glucose and insulin levels, 
Table 1. Characteristics of rabbits*

(Mean values with their standard errors)

\begin{tabular}{|c|c|c|c|c|c|c|c|c|c|c|c|c|}
\hline \multirow[b]{2}{*}{ Parameters } & \multicolumn{2}{|c|}{ CNT-5 $(n 6)$} & \multicolumn{2}{|c|}{ HFD-5 (n6) } & \multirow[b]{2}{*}{$P$} & \multicolumn{2}{|c|}{ CNT-10 $(n 6)$} & \multicolumn{2}{|c|}{ HFD-10 (n6) } & \multirow[b]{2}{*}{$P$} & \multicolumn{2}{|c|}{$P$} \\
\hline & Mean & SE & Mean & SE & & Mean & SE & Mean & SE & & $\begin{array}{l}\text { CNT-5 } v . \\
\text { CNT-10 }\end{array}$ & $\begin{array}{l}\text { HFD-5 } v \\
\text { HFD-10 }\end{array}$ \\
\hline Age $(d)$ & 125 & 5 & 131 & 2 & 0.287 & 149 & 2 & 153 & 4 & 0.446 & $<0.001$ & $<0.001$ \\
\hline Body weight $(\mathrm{kg})$ & 3.2 & 0.2 & 3.5 & 0.1 & 0.151 & 3.2 & 0.1 & 3.4 & 0.2 & 0.351 & 0.986 & 0.608 \\
\hline Plasma NEFA $(\mu \mathrm{mol} / \mathrm{ml})$ & 0.36 & 0.03 & 0.50 & 0.02 & 0.012 & 0.40 & 0.05 & 0.59 & 0.06 & 0.002 & 0.492 & 0.112 \\
\hline Ra of palmitate $(\mu \mathrm{mol} / \mathrm{kg}$ per $\min )$ & 4.8 & 0.1 & $4 \cdot 1$ & 0.3 & 0.367 & 4.4 & 0.8 & 3.7 & 0.3 & 0.372 & 0.548 & 0.547 \\
\hline Fasting glucose ( $\mathrm{mmol} / \mathrm{l})$ & $6 \cdot 11$ & 0.39 & 7.56 & 0.22 & 0.086 & 6.56 & 0.78 & $9 \cdot 17$ & 0.67 & 0.005 & 0.533 & 0.058 \\
\hline Serum insulin $(\mu \mathrm{U} / \mathrm{I})$ & $2 \cdot 1$ & 0.5 & 3.8 & 0.8 & 0.204 & $2 \cdot 2$ & $1 \cdot 1$ & 5.4 & $1 \cdot 2$ & 0.027 & 0.925 & 0.258 \\
\hline HOMA-IR & 0.51 & 0.15 & 1.28 & 0.28 & 0.199 & 0.80 & 0.46 & $2 \cdot 55$ & 0.60 & 0.008 & 0.621 & 0.042 \\
\hline HOMA- $\beta$ & 14 & 4 & 19 & 4 & 0.401 & 11 & 4 & 22 & 4 & 0.074 & 0.534 & 0.693 \\
\hline Disposition index & 0.04 & 0.01 & 0.07 & 0.01 & 0.139 & 0.05 & 0.02 & 0.12 & 0.02 & 0.004 & 0.468 & 0.023 \\
\hline Liver TAG $(\mu \mathrm{mol} / \mathrm{g})$ & 17 & 2 & 19 & 1 & 0.408 & 13 & 2 & 13 & 1 & 0.837 & 0.058 & 0.006 \\
\hline Liver NEFA $(\mu \mathrm{mol} / \mathrm{g})$ & $1 \cdot 15$ & 0.55 & 0.35 & 0.04 & 0.086 & 0.74 & 0.19 & $1 \cdot 31$ & 0.23 & 0.212 & 0.361 & 0.043 \\
\hline Muscle TAG $(\mu \mathrm{mol} / \mathrm{g})$ & $1 \cdot 7$ & 0.6 & 8.7 & 4.9 & 0.100 & 1.9 & 0.6 & $7 \cdot 3$ & $3 \cdot 2$ & $0 \cdot 217$ & 0.975 & 0.736 \\
\hline Muscle NEFA $(\mu \mathrm{mol} / \mathrm{g})$ & 0.15 & 0.03 & 0.11 & 0.02 & 0.542 & 0.15 & 0.05 & 0.22 & 0.06 & 0.268 & 0.939 & 0.081 \\
\hline \multicolumn{13}{|l|}{$\begin{array}{l}\text { Adipose tissue protein expression } \\
\text { (arbitrary units) }\end{array}$} \\
\hline t-PLIN & 3.09 & 0.13 & 3.49 & 0.09 & 0.215 & 3.20 & 0.34 & $2 \cdot 80$ & 0.24 & 0.225 & 0.736 & 0.040 \\
\hline p-PLIN:t-PLIN & 0.52 & 0.08 & 0.68 & 0.13 & 0.321 & 0.98 & 0.08 & 0.92 & 0.14 & 0.711 & 0.009 & 0.153 \\
\hline Adiponectin & 0.91 & 0.10 & 1.02 & 0.13 & 0.610 & $1 \cdot 12$ & 0.05 & 1.29 & 0.27 & 0.469 & 0.353 & 0.255 \\
\hline TLR4 expression & 0.27 & 0.04 & 0.29 & 0.04 & 0.650 & 0.20 & 0.02 & 0.27 & 0.03 & 0.177 & 0.125 & 0.515 \\
\hline
\end{tabular}

CNT, control; HFD, high-fat diet; Ra, rate of appearance; HOMA-IR, homeostatic model assessment of insulin resistance; t-PLIN, total perilipin; p-PLIN, phosphorylated perilipin; TLR4, toll-like receptor 4 .

* Differences were determined using two-way ANOVA with time and diet interaction with post hoc Tukey adjustment. $P<0.05$ was considered as statistically significant.

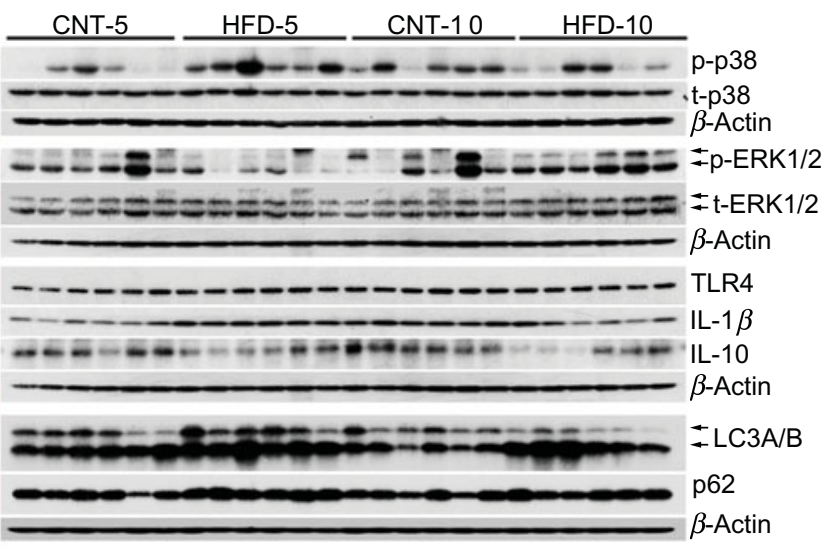

Fig. 1. Western blot images of subcutaneous adipose tissue samples. CNT, control; HFD, high-fat diet; p-p38, phosphorylated p38; t-p38, total p38; p-ERK1/2, phosphorylated ERK1/2; t-ERK1/2, total ERK1/2; TLR4, toll-like receptor 4; LC3A/B, microtubule-associated proteins $1 \mathrm{~A} / 1 \mathrm{~B}$ light chain 3 .

respectively, compared with CNT-5. The HFD-10 group had significantly higher levels of glucose $(P=0 \cdot 005)$, insulin $(P=0 \cdot 027)$, HOMA-IR $(P=0.008)$ and disposition index $(P=0.004)$ when compared with CNT-10. HOMA-IR $(P=0.042)$ and disposition index $(P=0.023)$ were also higher in HFD-10 when compared with the HFD-5 group; no differences were observed in HOMA- $\beta$ due to the dietary intervention at either time point (Table 1).

\section{Inflammatory pathways in peripheral blood mononuclear cells}

All data are presented in Fig. 2 and Table 2. HFD did not affect the expression of $\mathrm{t}-\mathrm{p} 38$, while the expression of $\mathrm{t}-\mathrm{ERK} 1 / 2$ significantly increased in HFD-10 when compared with CNT-10

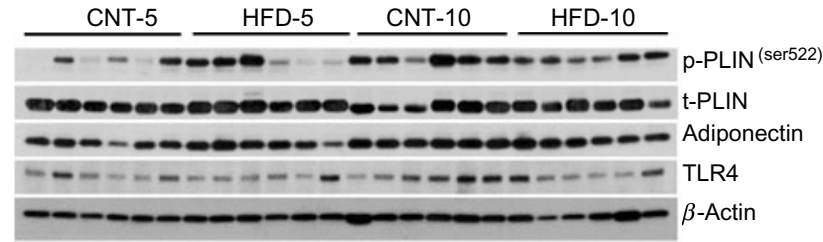

Fig. 2. Western blot images of peripheral blood mononuclear cell samples. CNT, control; HFD, high-fat diet; p-PLIN, phosphorylated perilipin; t-PLIN, total perilipin; TLR4, toll-like receptor 4 .

$(P=0.002)$. Compared with age-matched groups (i.e. CNT-5 and CNT-10, respectively), the activation of $\mathrm{p} 38$ (i.e. $\mathrm{p} / \mathrm{t}-\mathrm{p} 38$ ) significantly increased in HFD-5 group $(P<0.001)$ but decreased in HFD-10 group $(P=0 \cdot 028)$. The expression of $\mathrm{p}-\mathrm{ERK} 1 / 2$ significantly decreased in the HFD-5 group $(P=0 \cdot 008)$.

The expression of TLR 4 did not differ between the groups at any time point, while the expression of IL- $1 \beta$ significantly increased in HFD-5 $(P=0 \cdot 007)$. The expression of IL-10 significantly decreased in the HFD-10 group when compared with CNT-10 group $(P=0 \cdot 011)$. The IL-1 $\beta$ :IL-10 ratio was more than doubled in both HFD groups when compared with the corresponding CNT groups; however, the difference reached statistical significance at the 10 -week time point $(P=0 \cdot 011$; Table 2$)$.

\section{Autophagy pathways in peripheral blood mononuclear cells}

All data are presented in Fig. 2 and Table 2. The expression of microtubule-associated proteins $1 \mathrm{~A} / 1 \mathrm{~B}$ light chain 3 (LC3A/B-I) significantly decreased $(P=0.029)$ and the expression of LC3A/B-II significantly increased $(P=0.022)$ in the HFD-10 group when compared with the CNT-10. As a result, 
Table 2. Quantification of Western blot analyses of peripheral blood mononuclear cells (PBMC)*

(Mean values with their standard errors)

\begin{tabular}{|c|c|c|c|c|c|c|c|c|c|c|c|c|}
\hline \multirow[b]{2}{*}{$\begin{array}{l}\text { Parameters } \\
\text { (arbitrary units) }\end{array}$} & \multicolumn{2}{|c|}{ CNT-5 $(n 6)$} & \multicolumn{2}{|c|}{ HFD-5 (n6) } & \multirow[b]{2}{*}{$P$} & \multicolumn{2}{|c|}{ CNT-10 (n6) } & \multicolumn{2}{|c|}{ HFD-10 (n6) } & \multirow[b]{2}{*}{$P$} & \multicolumn{2}{|c|}{$P$} \\
\hline & Mean & SE & Mean & SE & & Mean & SE & Mean & SE & & $\begin{array}{l}\text { CNT-5 v. } \\
\text { CNT-10 }\end{array}$ & $\begin{array}{l}\text { HFD-5 } v \\
\text { HFD-10 }\end{array}$ \\
\hline$t-p 38$ & 0.56 & 0.03 & 0.63 & 0.04 & 0.143 & 0.56 & 0.03 & 0.60 & 0.03 & 0.411 & 0.985 & 0.514 \\
\hline p-p38:t-p38 & 0.82 & 0.23 & 2.33 & 0.28 & $<0.001$ & $2 \cdot 10$ & 0.33 & $1 \cdot 21$ & 0.21 & 0.028 & 0.003 & 0.008 \\
\hline t-ERK1/2 & 0.63 & 0.07 & 0.70 & 0.07 & 0.403 & 0.56 & 0.04 & 0.87 & 0.05 & 0.002 & 0.463 & 0.065 \\
\hline p-ERK $1 / 2: t-E R K 1 / 2$ & 3.53 & 0.42 & 1.21 & 0.23 & 0.008 & 3.44 & 0.94 & 2.90 & 0.34 & 0.499 & 0.915 & 0.044 \\
\hline TLR-4 & 0.43 & 0.02 & 0.49 & 0.03 & 0.143 & 0.42 & 0.04 & 0.44 & 0.02 & 0.601 & 0.866 & 0.258 \\
\hline IL-1 $\beta$ & 0.35 & 0.04 & 0.55 & 0.01 & 0.007 & 0.40 & 0.07 & 0.33 & 0.06 & 0.298 & 0.401 & 0.004 \\
\hline $\mathrm{IL}-10$ & $1 \cdot 10$ & 0.14 & 0.74 & 0.11 & 0.074 & $1 \cdot 10$ & 0.11 & 0.57 & 0.16 & 0.011 & 0.987 & 0.374 \\
\hline IL-1 $\beta: \mathrm{IL}-10$ & 0.33 & 0.05 & 0.83 & 0.13 & 0.060 & 0.38 & 0.07 & 0.95 & 0.32 & 0.011 & 0.868 & 0.636 \\
\hline LC3A/B-I & 0.38 & 0.05 & 0.50 & 0.04 & 0.054 & 0.38 & 0.04 & 0.25 & 0.04 & 0.029 & 0.913 & $<0.001$ \\
\hline LC3A/B-II & 0.67 & 0.06 & 0.80 & 0.02 & 0.054 & 0.58 & 0.04 & 0.74 & 0.04 & 0.022 & 0.155 & 0.314 \\
\hline LC3A/B-II/I & 1.87 & 0.30 & 1.68 & 0.16 & 0.572 & 1.55 & 0.08 & 3.21 & 0.33 & $<0.001$ & 0.357 & $<0.001$ \\
\hline p62 & 0.60 & 0.06 & 0.78 & 0.03 & 0.006 & 0.62 & 0.04 & 0.69 & 0.03 & 0.265 & 0.726 & 0.124 \\
\hline
\end{tabular}

CNT, control; HFD, high-fat diet; t-p38, total p38; p-p38, phosphorylated p38; t-ERK1/2, total ERK1/2; p-ERK1/2, phosphorylated ERK1/2; TLR4, toll-like receptor 4; LC3A/B, microtubule-associated proteins $1 \mathrm{~A} / 1 \mathrm{~B}$ light chain 3.

* Differences were determined using two-way ANOVA with time and diet interaction with post hoc Tukey adjustment. $P<0.05$ was considered as statistically significant.

Table 3. Acylcarnitine metabolism in peripheral blood mononuclear cells (PBMC)* (Mean values with their standard errors)

\begin{tabular}{|c|c|c|c|c|c|c|c|c|c|c|c|c|}
\hline \multirow[b]{2}{*}{ Parameter } & \multicolumn{2}{|c|}{ CNT-5 $(n 6)$} & \multicolumn{2}{|c|}{ HFD-5 $(n 6)$} & \multirow[b]{2}{*}{$P$} & \multicolumn{2}{|c|}{ CNT-10 (n6) } & \multicolumn{2}{|c|}{ HFD-10 $(n 6)$} & \multirow[b]{2}{*}{$P$} & \multicolumn{2}{|c|}{$P$} \\
\hline & Mean & SE & Mean & SE & & Mean & SE & Mean & $\mathrm{SE}$ & & $\begin{array}{l}\text { CNT-5 v. } \\
\text { CNT-10 }\end{array}$ & $\begin{array}{l}\text { HFD-5 } v \text {. } \\
\text { HFD-10 }\end{array}$ \\
\hline $\begin{array}{l}\text { Blood U-[}\left[{ }^{13} \mathrm{C}_{16}\right] \text {-palmitate enrichment } \\
\text { (MPE) }\end{array}$ & 8 & 2 & 14 & 2 & 0.064 & 12 & 1 & 15 & 2 & 0.335 & 0.216 & 0.744 \\
\hline Slope U-[13 $\left.\mathrm{C}_{16}\right]$-palmitoyl-carnitine & 0.016 & 0.001 & 0.050 & 0.001 & $<0.001$ & 0.038 & 0.001 & 0.032 & 0.001 & 0.505 & 0.021 & 0.055 \\
\hline FSR palmitoyl-carnitine (MPE/min) & 0.20 & 0.02 & 0.35 & 0.01 & $<0.001$ & 0.32 & 0.01 & 0.22 & 0.01 & 0.002 & $<0.001$ & $<0.001$ \\
\hline ASR palmitoyl-carnitine ( $\mathrm{nmol} / /$ per $\mathrm{min}$ ) & 0.047 & 0.003 & 0.059 & 0.004 & 0.060 & 0.056 & 0.006 & 0.043 & 0.002 & 0.048 & 0.138 & 0.020 \\
\hline Myristoyl-carnitine (nmol/l) & $2 \cdot 21$ & 0.29 & 1.50 & 0.27 & 0.054 & 1.85 & $0 \cdot 16$ & 1.57 & 0.26 & 0.453 & 0.309 & 0.845 \\
\hline Palmitoyl-carnitine $(\mu \mathrm{mol} / \mathrm{l})$ & 0.03 & 0.01 & 0.02 & 0.01 & 0.006 & 0.02 & 0.01 & 0.02 & 0.01 & 0.334 & 0.010 & 0.232 \\
\hline Total acylcarnitine $(\mu \mathrm{mol} / \mathrm{l})$ & 0.12 & 0.01 & 0.11 & 0.01 & 0.264 & 0.09 & 0.01 & 0.12 & 0.01 & 0.086 & 0.050 & 0.373 \\
\hline
\end{tabular}

CNT, control; HFD, high-fat diet; MPE, molar percentage excess; FSR, fractional synthesis rate; ASR, absolute synthesis rate.

* Differences were determined using two-way ANOVA with time and diet interaction with post hoc Tukey adjustment. $P<0.05$ was considered as statistically significant.

the ratio of LC3A/B-II/I significantly increased in the HFD-10 group when compared with the CNT-10 group as well as the HFD-5 group $(P<0.001)$. The expression of p62 increased in the HFD-5 group $(P=0.006)$ but remained unchanged in the HFD-10 group $(P=0.265)$ when compared with age-matched CNT groups.

\section{Acylcarnitine metabolism in peripheral blood mononuclear cells}

All data are presented in Table 3. The ex vivo FSR of palmitoylcarnitine was significantly higher in the HFD-5 group when compared with the CNT-5 group $(P<0.001)$ due to significantly higher slope of incorporation of the tracer into palmitoylcarnitine (i.e. the enrichment of $\mathrm{U}_{-}\left[{ }^{13} \mathrm{C}_{16}\right]$-palmitate-carnitine; $P<0.001)$. While in the HFD-10 group, the FSR of palmitoylcarnitine was significantly lower when compared with CNT-10 group $(P=0 \cdot 002)$. The ex vivo ASR of palmitoyl-carnitine tended to be higher in the HFD-5 group $(P=0.060)$ when compared with the CNT-5 group but significantly lower in the HFD-10 group compared with CNT-10 $(P=0.048)$ and HFD-5 $(P=0 \cdot 020)$ groups.

\section{Linear regression analyses}

The FSR of palmitoyl-carnitine was directly, though moderately, associated with the expression of p/t-p38 $(r 0.468, P=0.024)$, and IL-1 $\beta(r 0 \cdot 498, P=0 \cdot 015)$.

\section{Discussion}

Systemic low-grade inflammation is a main determinant of metabolic complications leading to the development of obesityrelated chronic diseases ${ }^{(22-24)}$. Thus, understanding the pathogenesis of this inflammation is critical in developing interventions to promote healthy living. Adipose tissue inflammation and the activation of circulating inflammatory cells are components of systemic low-grade inflammation ${ }^{(24)}$. However, the sequence of inflammatory events in the early stages of weight gain is still not well understood. Specifically, it is still unknown whether activation of inflammatory pathways in scAT precedes that of circulating immune cells (i.e. PBMC). Our results demonstrate that HFD induces an impairment in systemic substrate metabolism and activation of inflammatory and autophagy 
pathways in PBMC before metabolic and inflammatory changes occur in SCAT

High-fat diet-induced impaired systemic metabolism precedes changes in adipose tissue. HFD is well known to cause the development of metabolic abnormalities. Some, but not all, overfeeding studies have demonstrated that comparable periods of HFD resulted in significant increase in body weight and impaired tissue lipid metabolism ${ }^{(25-28)}$. In the present study, HFD did not significantly affect body weight, adipose tissue metabolism (i.e. Ra and activation of PLIN), inflammatory status (i.e. TLR4) or the deposition of lipids in liver and muscle (Table 1). This can potentially be explained by the differences in diet and fat composition ${ }^{(26)}$. The total and saturated fats in our study diet were approximately 21 and $10 \%$, respectively, amounts comparable to the average American daily intake ${ }^{(29)}$, but less than the $35 \%$ total and $12 \%$ saturated fats in a typical experimental Western-style diet ${ }^{(26)}$. Nevertheless, the HFD caused severe hyperfattyacidaemia in both 5 - and 10-week studies (Table 1). These data suggest that the scAT was in a state of adaptive expansion to accumulate the excess energy intake and that, under our study conditions, a 5-week or 10-week HFD feeding did not result in impaired tissue lipid metabolism with ectopic fat depositions. HFD led to worsening glycaemic control over time with the development of IR in HFD-10 group (Table 1), similar to other studies $^{(7,25,30)}$. In summary, a 5-week HFD impaired systemic lipid metabolism with increased levels of circulating NEFA, and a 10-week HFD impaired glycaemic control; however, these changes preceded the changes in muscle, liver and adipose tissue lipid metabolism as well as scAT inflammatory status.

\section{High-fat diet-induced activation of inflammatory pathways} in peripheral blood mononuclear cells. The main aim of the present study was to determine the effect of HFD on activation of inflammatory pathways in PBMC. Previous studies showed activation of mitogen-activated protein kinase (MAPK; i.e. p38 and ERK1/2) pathways in lymphocytes and monocytes of patients with type 2 diabetes ${ }^{(31-33)}$. In experimental models, specific inhibition of MAPK pathways in macrophages suppressed pro-inflammatory responses with delayed onset of diabetes ${ }^{(34)}$. Therefore, the MAPK pathway is important in obesityrelated inflammation. Indeed, after 5 weeks of HFD, a significant activation of $\mathrm{p} 38$ (i.e. $\mathrm{p} / \mathrm{t}-\mathrm{p} 38$ ) and increased expression of IL- $1 \beta$ were observed (Fig. 2, Table 2), though the ERK1/2 was either decreased in HFD-5 or unaffected in HFD-10. Both p38 and ERK1/2 are involved in the MAPK pathway, and p38 is strongly associated with inflammation but ERK1/2 with cell differentiation pathways ${ }^{(35)}$. To determine the mechanisms of MAPK pathway activation, we measured the expression of TLR 4 and IL- $1 \beta$. TLR4, a member of the pattern-recognition receptor family TLR, is a well-known receptor involved in SFA-induced activation of inflammatory pathways including MAPK pathways ${ }^{(36,37)}$. The expression of TLR4 did not differ between groups at any time $(P>0.05$; Fig. 2, Table 2$)$. IL- $1 \beta$ is also known to activate the MAPK pathways ${ }^{(38)}$. Indeed, the expression of IL- $1 \beta$ followed the pattern of changes in expression of $\mathrm{p} / \mathrm{t}-\mathrm{p} 38$ and was significantly increased only in HFD-5 (Fig. 2, Table 2). These data may suggest that HFD caused inflammatory changes in the PBMC of rabbits via IL-1 $\beta$ but not TLR4-involved mechanisms. However, these changes occurred after short-term (i.e. 5 weeks) HFD only, because the expression of both p-p38 and IL- $1 \beta$ returned to CNT-5 levels in the HFD-10 group (Fig. 2, Table 2). This may suggest that either adaptive mechanisms combating the inflammatory changes are being activated or other changes, yet unknown, are occurring that result in the 'quiescence' of inflammatory response at 10 weeks of HFD. The expression of IL-10, a potent anti-inflammatory cytokine ${ }^{(39)}$, was $32 \%$ lower in the HFD-5 group than that in CNT-5 $(P=0.074)$, and $48 \%$ lower in the HFD-10 group than that in CNT-10 group $(P=0 \cdot 011$; Fig. 2, Table 2). These data may suggest that IL-10 may not be involved in suppression of inflammation in PBMC in the HFD10 group. However, IL-10 is the classical M2 or immune regulatory cytokine, while IL- $1 \beta$ is the classical M1 or inflammatory type ${ }^{(21,39)}$. Thus, HFD may lead to the loss of M2-type immune cells. As a result, although IL- $1 \beta$ did not differ between the HFD-10 and CNT-10 groups, the higher IL-1 $\beta$ :IL-10 ratio suggests that HFD-10 animals were immune compromised. In summary, short-term HFD (i.e. HFD-5 group) induced activation of inflammatory pathways in PBMC in vivo in rabbits and ultimately immune deficiency in the HFD-10 group.

High-fat diet-modified autophagy pathways in peripheral blood mononuclear cells. Autophagy plays a housekeeping role in clearing misfolded or aggregated proteins and damaged organelles and has also been shown to suppress excessive inflammation $^{(40)}$. Activation of microtubule-associated proteins 1A/1B light chain 3 (LC3), a central protein in the autophagy pathway $^{(41)}$, is the main step in monocyte-macrophage proinflammatory differentiation ${ }^{(13)}$. Upon autophagic signal, the LC3A/B-I isoform undergoes lipidation to form the LC3A/B-II isoform, which shows faster electrophoretic mobility in SDSPAGE gels and thus shows up at $14-16 \mathrm{kDa}$, in comparison with $16-18 \mathrm{kDa}$ for LC3A/B-I. The increased ratio of LC3-II:LC3-I is a marker for autophagosomes formation. LC3A/B-II/I was significantly increased in PBMC of the HFD-10 group (Fig. 2, Table 2), suggesting the formation of autophagosomes. Thus, these data may suggest that autophagosomes were formed as a response to activation of inflammatory pathways. However, the autophagy process is comprised of autophagosome formation, fusion of autophagosomes with lysosomes into autophagolysosomes and the maturation of this complex for degradation or sequestration. p62 is an autophagy substrate that delivers ubiquitinated cargoes for autophagic degradation and is used as a reporter of autophagy activity. Active degradation of autophagolysosomes reduces p62 expression, while unchanged or increased p62 expression, along with increased LC3A/B-II/I, suggesting modification of the autophagy pathway ${ }^{(41)}$. Here p 62 expression increased in the HFD-5 group but unchanged in the HFD-10 group (Fig. 2, Table 2). These data demonstrate that HFD induced a modified autophagy in PBMC in vivo. Increased expression of both LC3A/B-II and p62 is critical in GM-CSFinduced monocyte-macrophage differentiation, suggesting that 
this modified autophagy pathway is crucial in this process ${ }^{(13)}$. In obese mice, impaired macrophage autophagy enhanced pro-inflammatory monocyte-macrophage polarisation ${ }^{(42)}$. RostamiRad et al. also showed that treating PBMC with palmitate significantly increases LC3A/B and p62 expressions ${ }^{(12)}$. In summary, our data suggest that a 10-week HFD induced modified autophagy in PBMC in vivo in rabbits.

Acylcarnitine metabolism in peripheral blood mononuclear cells. The activation of inflammatory pathways can be caused by intermediate lipid metabolites (i.e. acylcarnitines), which we previously reported were produced in PBMC using NEFA ${ }^{(16)}$. We also demonstrated that the ASR of palmitoyl-carnitine, estimated during a hyperinsulinaemic-euglycaemic clamp, inversely correlates with the degree of systemic insulin sensitivity in healthy overweight women. Therefore, we measured the ex vivo FSR and ASR of palmitoyl-carnitine by blood cells ${ }^{(16)}$. The ex vivo FSR of palmitoyl-carnitine was significantly higher in the HFD- 5 group than in the CNT- 5 group but significantly lower in the HFD-10 group than in the CNT-10 group. The mechanism of adaptation to an HFD or high-energy intake is an increase in fat mass and/or in FAO until a new equilibrium is reached to accommodate the intake ${ }^{(43)}$. However, in an IR or metabolically unhealthy state, the FAO is lower and cannot accommodate the excess energy intake ${ }^{(44)}$. Thus, our data on palmitoyl-carnitine synthesis in PBMC follow this pattern of adaptive FAO with HFD intake. Interestingly, the linear regression analyses demonstrated a direct, albeit moderate, correlation of acylcarnitine metabolism with $\mathrm{p} / \mathrm{t}-\mathrm{p} 38$ and IL-1 $\beta$ expression. Therefore, p38 activation and increased IL-1 $\beta$ expression may result from HFD-induced enhanced FAO as estimated by the ex vivo FSR of palmitoyl-carnitine. Vice versa, the lower FSR in the HFD-10 group may explain the 'quiescence' of inflammatory pathways or the switch inducing the modified autophagy.

\section{Study limitations}

(1) For the HFD group, a chow diet was supplemented with $18 \%$ fat. Thus, the proportion of nutrients, not only fats, was different between the diet (supplemental file). In our future studies, we may consider using dietary approaches that differ in composition of macronutrients but not of other nutrients. In general, rabbits' food of choice contains low fats. However, rabbits fed HFD or high-cholesterol diet develop impaired fat metabolism like that of humans with obesity ${ }^{(14)}$ or typical characteristics of atherosclerosis $^{(45)}$, respectively. Thus, we think that the HFD used in the present study is acceptable to allow the present conclusions to be drawn. (2) We did not study VAT in the present study. The currently available data suggest that scAT ability to accumulate lipids is instrumental in averting the expansion of VAT in early stages of weight gain as well as in the development of obesityassociated metabolic abnormalities ${ }^{(8,17,25,46,47)}$. However, we plan to study VAT in the future. (3) Our study lacks interventions to determine whether averting the changes in FAO in PBMC prevents the activation of inflammatory and autophagy pathways, which will be addressed in future studies.

\section{Conclusion}

Our data confirm that HFD caused hyperfattyacidaemia, peripheral IR, enhanced FAO and activation of inflammatory and autophagy pathways in PBMC, without changes in scAT metabolism and inflammation. These outcomes are similar to those observed in humans with obesity. The time course of these changes was not straightforward, with some occurring after 5 weeks and others after 10 weeks of HFD. The adaptive metabolic and anti-inflammatory response to the diet may be the main explanations for our findings. (a) Excessive FAO causes a domino effect, activating inflammatory and autophagy pathways in PBMC and subsequent systemic low-grade adipose tissue inflammation and (b) if dietary antioxidants correlate with a better health outcome, our findings may explain why, within a group with comparable BMI, some are metabolically healthier than others ${ }^{(48,49)}$. Although the detailed mechanism is not yet characterised, we hypothesise that dietary hyperfattyacidaemia causes enhanced FAO-induced activation of p38 in PBMC, which may induce the activation of autophagy pathways. Alternatively, IR or an impaired glycaemic control-associated decline in FAO (HFD-10) activated the autophagy pathways. Because (a) impaired activation of autophagy pathways plays a crucial role in monocyte-macrophagy differentiation and (b) the Western lifestyle is characterised by a continuous postprandial state, PBMC undergoing cycles of activation of inflammatory/autophagy pathways may be the metabolic mechanism linking HFD to systemic low-grade inflammation. Thus, interventions to avert HFD-induced early activation of inflammatory and modified autophagy pathways in PBMC could help to prevent metabolic and inflammatory complications of obesity and promote healthier living.

\section{Acknowledgements}

We thank Christopher Danesi, Kathleen Randolph and Anahi D. Delgadillo for help with sample analyses. We thank Dr Sarah Toombs-Smith for the critical editing of this work.

The present study was supported by the 1KL2TR001441 NIH Training grant, the Institute for Translational Sciences at the UTMB, and in part by a Clinical and Translational Science Award (no. UL1 TR001439) from the National Center for Advancing Translational Sciences, National Institutes of Health, and the Shriners Grant no. 84090, Metabolism Unit, Shriners Hospitals for Children.

D. R. T. was responsible for hypothesis generation and securing funds. J. E. L., C. A., E. M., D. L. B. and D. R. T. conducted the study. B. T., T. J. W., E. L. D., T. B. S., C. P., J. J. E. and D. R. T. analysed the samples. J. E. L., B. T., T. J. W., E. L. D., T. B. S., C. P., J. J. E., M. S.-M., E. V., R. F., N. A. and D. R. T. analysed and interpreted the data. C. R. A. and D. R. T. performed the statistical analyses. All contributors were responsible for manuscript writing, editing and final approval.

The authors declare no competing financial interests.

\section{Supplementary material}

To view supplementary material for this article, please visit https://doi.org/10.1017/S0007114519002472 


\section{References}

1. Ghanim H, Aljada A, Hofmeyer D, et al. (2004) Circulating mononuclear cells in the obese are in a proinflammatory state. Circulation 110, 1564-1571.

2. Viardot A, Heilbronn LK, Samocha-Bonet D, et al. (2012) Obesity is associated with activated and insulin resistant immune cells. Diabetes Metab Res Rev 28, 447-454

3. Bañuls C, Rovira-Llopis S, Lopez-Domenech S, et al. (2017) Oxidative and endoplasmic reticulum stress is impaired in leukocytes from metabolically unhealthy vs healthy obese individuals. Int J Obes (Lond) 41, 1556-1563.

4. Wensveen FM, Valentić S, Šestan M, et al. (2015) The "Big Bang" in obese fat: events initiating obesity-induced adipose tissue inflammation. Eur J Immunol 45, 2446-2456.

5. Pahlavani M, Ramalho T, Koboziev I, et al. (2017) Adipose tissue inflammation in insulin resistance: review of mechanisms mediating anti-inflammatory effects of omega-3 polyunsaturated fatty acids. J Investig Med 65, 1021-1027.

6. Permana PA, Menge C \& Reaven PD (2006) Macrophagesecreted factors induce adipocyte inflammation and insulin resistance. Biochem Biophys Res Commun 341, 507-514.

7. Tam CS, Viardot A, Clément K, et al. (2010) Short-term overfeeding may induce peripheral insulin resistance without altering subcutaneous adipose tissue macrophages in humans. Diabetes 59, 2164-2170.

8. Alligier M, Gabert L, Meugnier E, et al. (2013) Visceral fat accumulation during lipid overfeeding is related to subcutaneous adipose tissue characteristics in healthy men. J Clin Endocrinol Metab 98, 802-810.

9. Ehlers K, Brand T, Bangert A, et al. (2014) Postprandial activation of metabolic and inflammatory signalling pathways in human peripheral mononuclear cells. Br J Nutr 111, $2167-2175$.

10. Gill JM \& Hardman AE (2003) Exercise and postprandial lipid metabolism: an update on potential mechanisms and interactions with high-carbohydrate diets (review). I Nutr Biochem 14, 122-132.

11. Henson J, Dunstan DW, Davies MJ, et al. (2016) Sedentary behavior as a new behavioral target in the prevention and treatment of type 2 diabetes. Diabetes Metab Res Rev 32 , Suppl. 1, 213-220.

12. RostamiRad A, Ebrahimi SSS, Sadeghi A, et al. (2018) Palmitateinduced impairment of autophagy turnover leads to increased apoptosis and inflammation in peripheral blood mononuclear cells. Immunobiology 223, 269-278.

13. Zhang Y, Morgan MJ, Chen K, et al. (2012) Induction of autophagy is essential for monocyte-macrophage differentiation. Blood 119, 2895-2905.

14. Zhang XJ, Chinkes DL, Aarsland A, et al. (2008) Lipid metabolism in diet-induced obese rabbits is similar to that of obese humans. J Nutr 138, 515-518.

15. Zhang XJ, Wang L, Tuvdendorj D, et al. (2013) Acute hyperinsulinemia and reduced plasma free fatty acid levels decrease intramuscular triglyceride synthesis. Metabolism 62 , $44-51$.

16. Chondronikola M, Asghar R, Zhang X, et al. (2017) Palmitoylcarnitine production by blood cells associates with the concentration of circulating acyl-carnitines in healthy overweight women. Clin Nutr 36, 1310-1319.

17. Pan W, Ciociola E, Saraf M, et al. (2011) Metabolic consequences of ENPP1 overexpression in adipose tissue. $A m J$ Physiol Endocrinol Metab 301, E901-E911.

18. Gonzales CM, Williams CB, Calderon VE, et al. (2015) Antibacterial role for natural killer cells in host defense to Bacillus anthracis. Infect Immun 80, 234-242.
19. Matthews DR, Hosker JP, Rudenski AS, et al. (1985) Homeostasis model assessment: insulin resistance and beta-cell function from fasting plasma glucose and insulin concentrations in man. Diabetologia 28, 412-419.

20. Matsuda M (2010) Measuring and estimating insulin resistance in clinical and research settings. Nutr Metab Cardiovasc Dis 20, 79-86.

21. Jamil B, Shahid F, Hasan Z, et al. (2007) Interferon gamma/IL10 ratio defines the disease severity in pulmonary and extra pulmonary tuberculosis. Tuberculosis (Edinb) 87, 279-287.

22. Shoelson SE, Herrero L \& Naaz A (2007) Obesity, inflammation, and insulin resistance. Gastroenterology 132, 2169-2180.

23. Xu H, Barnes GT, Yang Q, et al. (2003) Chronic inflammation in fat plays a crucial role in the development of obesity-related insulin resistance. J Clin Invest 112, 1821-1830.

24. Hotamisligil GS (2017) Inflammation, metaflammation and immunometabolic disorders. Nature 542, 177-185.

25. Cuthbertson DJ, Steele T, Wilding JP, et al. (2017) What have human experimental overfeeding studies taught us about adipose tissue expansion and susceptibility to obesity and metabolic complications? Int J Obes (Lond) 41, 853-865.

26. Lionetti L, Mollica MP, Sica R, et al. (2014) Differential effects of high-fish oil and high-lard diets on cells and cytokines involved in the inflammatory process in rat insulin-sensitive tissues. Int J Mol Sci 15, 3040-3063.

27. Sevastianova K, Santos A, Kotronen A, et al. (2012) Effect of short-term carbohydrate overfeeding and long-term weight loss on liver fat in overweight humans. Am J Clin Nutr 96, 727-734.

28. von Frankenberg AD, Marina A, Song X, et al. (2017) A high-fat, high-saturated fat diet decreases insulin sensitivity without changing intra-abdominal fat in weight-stable overweight and obese adults. Eur J Nutr 56, 431-443.

29. Centers for Disease Control and Prevention. National Health and Nutrition Survey. Intake of calories and selected nutrients for the United States population, 1999-2010. https://www.cdc. gov/nchs/data/nhanes/databriefs/calories.pdf (accessed October 2019).

30. Koopman KE, Caan MW, Nederveen AJ, et al. (2014) Hypercaloric diets with increased meal frequency, but not meal size, increase intrahepatic triglycerides: a randomized controlled trial. Hepatology 60, 545-553.

31. Tchaikovski V, Olieslagers S, Böhmer FD, et al. (2009) Diabetes mellitus activates signal transduction pathways resulting in vascular endothelial growth factor resistance of human monocytes. Circulation 120, 150-159.

32. He L, Wong CK, Cheung KK, et al. (2013) Anti-inflammatory effects of exendin- 4 , a glucagon-like peptide- 1 analog, on human peripheral lymphocytes in patients with type 2 diabetes. J Diabetes Investig 4, 382-392.

33. Olieslagers S, Pardali E, Tchaikovski V, et al. (2011) TGF- $\beta 1 /$ ALK5-induced monocyte migration involves PI3K and p38 pathways and is not negatively affected by diabetes mellitus. Cardiovasc Res 91, 510-518.

34. Jeong HW, Hsu KC, Lee JW, et al. (2009) Berberine suppresses proinflammatory responses through AMPK activation in macrophages. Am J Physiol Endocrinol Metab 296, E955-E964.

35. Johnson GL \& Lapadat R (2002) Mitogen-activated protein kinase pathways mediated by ERK, JNK, and p38 protein kinases. Science 298, 1911-1912.

36. Jialal I, Kaur H \& Devaraj S (2014) Toll-like receptor status in obesity and metabolic syndrome: a translational perspective. J Clin Endocrinol Metab 99, 39-48.

37. Kochumon S, Wilson A, Chandy B, et al. (2018) Palmitate activates CCL4 expression in human monocytic cells via 
TLR4/MyD88 dependent activation of NF-кB/MAPK/ PI3K signaling systems. Cell Physiol Biochem 46, 953-964.

38. Kulawik A, Engesser R, Ehlting C, et al. (2017) IL-1 $\beta$-induced and p38MAPK-dependent activation of the mitogenactivated protein kinase-activated protein kinase 2 (MK2) in hepatocytes: signal transduction with robust and concentration-independent signal amplification. $J$ Biol Chem 292, 6291-6302.

39. Bazzoni F, Tamassia N, Rossato M, et al. (2010) Understanding the molecular mechanisms of the multifaceted IL-10-mediated anti-inflammatory response: lessons from neutrophils. Eur J Immunol 40, 2360-2368.

40. Zhong Z, Umemura A, Sanchez-Lopez E, et al. (2016) NF-кB restricts inflammasome activation via elimination of damaged mitochondria. Cell 164, 896-910.

41. Klionsky DJ, Abdelmohsen K, Abe A, et al. (2016) Guidelines for the use and interpretation of assays for monitoring autophagy (3rd edition). Autophagy 12, 1-222.

42. Liu K, Zhao E, Ilyas G, et al. (2015) Impaired macrophage autophagy increases the immune response in obese mice by promoting proinflammatory macrophage polarization. Autophagy 11, 271-284.
43. Koves TR, Ussher JR, Noland RC, et al. (2008) Mitochondrial overload and incomplete fatty acid oxidation contribute to skeletal muscle insulin resistance. Cell Metab 7, 45-56.

44. Kelley DE, Goodpaster B, Wing RR, et al. (1999) Skeletal muscle fatty acid metabolism in association with insulin resistance, obesity, and weight loss. Am J Physiol 277, E1130-E1141.

45. Taylor E, Huang N, Bodde J, et al. (2018) MRI of atherosclerosis and fatty liver disease in cholesterol fed rabbits. J Transl Med 16, 215.

46. Tuvdendorj D, Chandalia M, Batbayar T, et al. (2013) Altered subcutaneous abdominal adipose tissue lipid synthesis in obese, insulin-resistant humans. Am J Physiol Endocrinol Metab 305, E999-E1006.

47. Abate N \& Chandalia M (2017) Risk of obesity-related cardiometabolic complications in special populations: a crisis in Asians. Gastroenterology 152, 1647-1655.

48. Mongraw-Chaffin M, Foster MC, Anderson CAM, et al. (2018) Metabolically healthy obesity, transition to metabolic syndrome, and cardiovascular risk. J Am Coll Cardiol 71, 1857-1865.

49. Guo F \& Garvey WT (2016) Cardiometabolic disease risk in metabolically healthy and unhealthy obesity: stability of metabolic health status in adults. Obesity (Silver Spring) 24, 516-525. 